\title{
Application of laparoscopy in acute appendicitis for the elderly: a single center experience.
}

\author{
Yunshan Duan ${ }^{1 \#, ~ H o n g j u n ~ Y a n g ~}{ }^{2 \#}$, Yapeng Yang', Guang Wang ${ }^{1}$, Tangshun Wang ${ }^{1}$, Zhenzhou Chen ${ }^{1}$, \\ Xiang Gao ${ }^{1}$, Tao Jiang ${ }^{*}$, Xiaoguang Shi ${ }^{{ }^{*}}$ \\ ${ }^{1}$ Department of General Surgery, Dongzhimen Hospital, the First Affiliated Hospital of Beijing University of Chinese \\ Medicine, Beijing, PR China
}

${ }^{2}$ Department of General Surgery, Beijing Jingmei Group General Hospital, Beijing, PR China

\#These authors contributed equally to this work

\begin{abstract}
Background and purpose: Laparoscopic appendectomy for acute appendicitis is gold standard for adult patients in the world. The incidence of appendicitis in the elderly has risen. The aim of this study was to compare post-operatory outcomes in old patients with acute appendicitis approached by laparoscopy versus open surgery.

Methods: We retrospectively analysed the data of elderly patients who underwent appendectomy for acute appendicitis from $1^{\text {st }}$ of January 2010 to the $1^{\text {st }}$ of August 2017. We analysed 286 appendectomies in elderly patients: 214 patients were operated by laparoscopic appendectomy (LA) and 72 patient were underwent Open Appendectomy (OA).

Results: The operation time was slightly longer in the LA group $(\mathbf{P}<0.001)$, but the time of recovery inflammatory response, time for diet and hospital stay were significantly shorter in LA group than that in OA group. Complications: the rate of surgical site infection in LA group was less than that in OA group, and the rate of pneumonia between LA group and OA group was similar.

Conclusions: Laparoscopic appendectomy can be recommended as first choice for old patients with appendicitis.
\end{abstract}

Keywords: Laparoscopic appendectomy, Open appendectomy, Acute appendicitis, Elderly.

Accepted on February 22, 2018

\section{Introduction}

Appendicitis is still the most common emergency surgical condition in the world [1], and the incidence of acute appendicitis in elderly patients aged more than $65 \mathrm{y}$ was about $10 \%$ [2]. With the greatly increasing in life expectancy, more elderly patients are being diagnosed with acute appendicitis and the outcomes are poor because of long time intervals to diagnosis and treatment [3]. Since laparoscopy was first described by Semm in 1983 [4], this technology has widely applied in the diagnosis and treatment of many elective and emergency surgeries. Due to several advantages, such as less tissue trauma, earlier recovery of intestinal motility, less adhesions and shorter hospitalization, some doctors even suggest laparoscopy as gold standard for acute appendicitis [5]. AS the elderly patients with deteriorating organs have lower physiological reserve than the young adults, it is controversial that laparoscopic appendectomy could be recommended for the old patients with acute appendicitis. The aim of this analysis is to evaluate whether elderly patients with acute appendicitis could benefit from laparoscopic appendectomy comparing with open appendectomy.

\section{Materials and Methods}

We retrospectively analysed the elder patient records of all patients who were 65 years or more and underwent appendectomies at our institution between January 2010 and August 2017. This study was approved by the Ethical Committee of Dongzhimen Hospital, Beijing University of Chinese Medicine. The diagnosis of appendicitis before operation mainly depended on the medical history and the clinical examination: pain to the right lower quadrant or rebound tenderness, fever, elevated White Blood Cell count (WBC) and elevated C Reactive Protein (CRP), and Ultrasonographic (US) or Computer Tomographic (CT) findings of the abdomen [6]. In total, 286 patients with postoperative diagnosis of appendicitis were included and 38 patients were excluded with absence of histological confirmation of appendicitis or with other abdominal illness. 214 patients were operated by Laparoscopic Appendectomy 
(LA) and 72 patient were underwent Open Appendectomy (OA).

All surgeries were performed by an attending doctor and a senior resident of the department of general surgery of our hospital. The surgical approach was followed by surgeon's preference and criteria. LA were performed using a three trocar technique: two $10 \mathrm{~mm}$ and one $5 \mathrm{~mm}$ ports. The mesoappendix was transected with ultrasonic energy or homopolar coagulation. The appendix was ligated in the base with hem-olock clip or intracorporeal knotting and extraction was made using a plastic bag. A drain tube was placed in case of perforation and abscesses. For OA, a 5-8 cm McBurney's incision was made in the right lower abdominal quadrant, and appendectomy was followed by tying off of the mesoappendix and radix of the appendix. The appendix stump was embedded. A drain was used in the rectovesical area when considered necessary. All patients received with cefuroxime sodium in combination with metronidazole or etimicin sulfate in case of allergies as perioperative antibiotic prophylaxis.

The following clinical variables were recorded: patients' sex, ages, comorbidities, American Society of Anesthesiology (ASA) score, operative time, time of antibiotics using, time for diet, hospitalization time, complication rate, and other parameters.

And a quality of life questionnaire of operated patients was required to be completed via telephone 1 month after discharge of the patients from our hospital [7]. Some questions, such as satisfaction with the size and appearance of the scar(s), the quality and intensity of the postoperative pain, were asked to be completed. The answers were evaluated using 4 point scores: not at all $=1$, few $=2$, moderate $=3$ and very $=4$. A control question was asked twice in order to ensure the reliability of the answers.

Descriptive and comparative statistical analyses were performed using Statistical Software for the Social Sciences (SPSS) version 19 (SPSS Inc., Chicago, IL, USA). Continuous variables are presented as (means \pm standard deviations) and were compared between groups with Student's t test or Mann Whitney test, as appropriate by type of distribution. Categorical variables were compared with chi-square test or Fisher exact test, depending on the number of observations. For the comparison of the scores of SF-36, a Wilcoxon rank sum test was applied. A $\mathrm{P}$ value of $<0.05$ was considered significant.

\section{Results}

Two hundred and eighty six patients with post-operative diagnosis of appendicitis were identified, 214 of them were treated by Laparoscopic Appendectomy (LA) and 72 patients were underwent Open Appendectomy (OA). There were no difference in mean age, gender, comorbidities, ASA score, WBC count and CRP before operation between LA group and OA group (Table 1).
Laparoscopic appendectomy showed a longer operative time $(\mathrm{P}<0.001)$ than open appendectomy but a shorter time of duration of antibiotics use, diet and hospital stay $(\mathrm{P}<0.001)$ as compared to OA (Table 2). And the operation bleeding, postoperative bleeding and the cases of cecal fistula of LA group were all less than that of OA group. WBC count of POD 3 showed the patients receiving LA made earlier recovery than those receiving OA. Although 3 patients of LA group appeared subcutaneous emphysemasurgical site infection rate of LA was significant lower than that of OA. And 3 patients of OA group showed urinary retention after operation. There were no differences in pneumonia between LA group and OA group (Table 2). Evaluation of the appearance of the scar(s) and the postoperative pain showed that significantly more patients of the LA group were satisfied with the method of laparoscopy than the OA group (Figure 1a, $\mathrm{P}<0.001$; Figure 1b, $\mathrm{P}<0.001$ ).
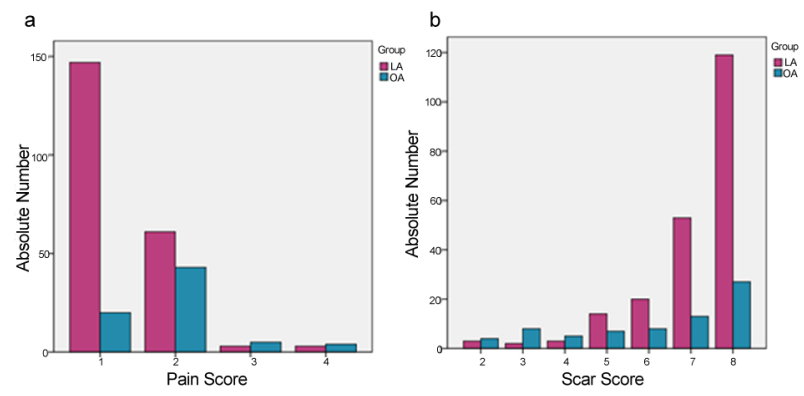

Figure 1. Postoperative pain and satisfaction. a: Dull pain in the abdomen; 1: not at all, 2: few, 3: moderate, 4: very; $b$ : Postoperative satisfaction with appearance and size of the scar: 2: minimal satisfaction, 8: maximal satisfaction.

Table 1. Demographic and preoperative data in 286 old patients with appendicitis.

\begin{tabular}{llll}
\hline Variables & LA $(\mathbf{n = 2 1 4})$ & OA $(\mathbf{n}=\mathbf{7 2})$ & P value \\
\hline Age (median \pm SD) & $68.48 \pm 7.32$ & $70.16 \pm 6.67$ & 0.314 \\
\hline Gender (\%) & $136(63.6 \%)$ & $39(54.2 \%)$ & 0.159 \\
\hline Male & $78(36.4 \%)$ & $33(45.8 \%)$ & \\
\hline Female & $153(71.5 \%)$ & $56(77.8 \%)$ & 0.299 \\
\hline Comorbidities (\%) & $14.62 \pm 4.86$ & $13.57 \pm 5.15$ & 0.31 \\
\hline WBC count (109/L) (median \pm & & \\
SD) & & & \\
\hline ASA score (\%) & $61(28.5)$ & $16(22.2)$ & 0.176 \\
\hline I & $153(71.5)$ & $54(75.0)$ & \\
\hline II & 0 & $2(2.8)$ & 0.891 \\
\hline III & 0 & $38(52.8)$ & \\
\hline IV & $104(48.6)$ & & \\
\hline Localized peritonitis (\%) & & & \\
\hline
\end{tabular}

Table 2. Operative characteristics and postoperative parameters in 286 old patients with appendicitis. 


\begin{tabular}{llll}
\hline Variables & LA (n=214) & OA (n=72) & P value \\
\hline Operative time (min) (median \pm SD) & $68 \pm 27$ & $52 \pm 35$ & $<0.001$ \\
\hline Bleeding (ml) & $5.4 \pm 4.3$ & $10.5 \pm 4.3$ & $<0.001$ \\
\hline Postoperative bleeding (case) & 1 & 3 & 0.021 \\
\hline Cecal fistula & 3 & 8 & $<0.001$ \\
\hline WBC count (109/L) POD 1 & $11.38 \pm 4.21$ & $12.11 \pm 3.86$ & 0.12 \\
\hline WBC count (109/L) POD 3 & $6.95 \pm 3.35$ & $8.26 \pm 4.64$ & $<0.001$ \\
\hline Time for diet (day) & $1.31 \pm 2.24$ & $2.87 \pm 3.10$ & $<0.001$ \\
\hline Surgical site infection (\%) & $4(1.87)$ & $7(9.72)$ & 0.007 \\
\hline Urinary retention (\%) & 0 & $3(4.17)$ & 1 \\
\hline Subcutaneous emphysema (\%) & $3(1.40)$ & 0 & 1 \\
\hline Pneumonia (\%) & $1(0.47)$ & $2(2.78)$ & 0.157 \\
\hline Intra-abdominal abscess & 0 & 1 & 1 \\
\hline Duration of antibiotics use (day) & $3.58 \pm 2.24$ & $5.14 \pm 3.42$ & $<0.001$ \\
\hline Prolonged ileus (\%) & 3 & 5 & 0.014 \\
\hline Hospitalization time (day) & $4.52 \pm 2.89$ & $6.17 \pm 3.67$ & $<0.001$ \\
\hline
\end{tabular}

complication in the elderly, we most concerned, in the LA group, such as pneumonia, was similar to the rate in the OA group. During the LA operation, we tended to decrease the pressure of laparoscopic pneumoperitoneum to $10 \mathrm{mmHg}$ to relieve the heart and lungs' burden. During the general anesthesia, the anesthesiologist tried to maintain a stable blood pressure and avoids the fluctuation of the patient's blood pressure, especially when we were establishing the pneumoperitoneum. Although LA took little longer operation time than OA in elderly, we believed the benefit of old patients was worth choosing LA, and this operation time gap between LA and OA would gradually decrease with the proficiency in laparoscopic surgery. Moreover, the patients' quality of life after the laparoscopic appendectomy may be another important factor to be taken into account for the patients and the scholars. Appendectomy is one of the most frequently performed emergency operations. So, we mainly concerned two aspects: appearance of the scar(s) and the discomforts after operations. The results were very interesting: the patients of LA group showed better felling than patients of OA group. And the old patients were also concerned about the appearance of the incisions than assumed. A significantly higher satisfaction of the patients in the laparoscopic group concerning size and appearance of scars. So the long term results about Appendectomy should be taken count in during comparison of LA vs. OA.

Taken together, the findings of this study demonstrated that laparoscopic approach is a safe and suitable approach to the old patients with appendicitis. Laparoscopic appendectomy for is going to the first choice for acute appendicitis in the elderly.

\section{Conflict of Interest}

The authors declare that they have no conflict of interest. to often vague symptoms, blunted tenderness, and diminished leukocytic responses [3]. And the delay in seeking medical care and in making diagnosis makes the inflammation progress in the elderly more serious than in the young patients. Direct exploration of the abdominal viscera is one of many advantages of laparoscopy in old patients, particularly in nondiagnostic or complicated cases. In our study, 38 patients were found to have other illness and received proper treatment benefitting from this viewable exploration. Laparoscopy also shows the low rates of postoperartive adhesion and mechanical bowel obstruction $[9,10]$. In our study, LA group showed shorter time for diet and less rate prolonged ileus than those of OA group. The reasons mainly depend on LA could examine the whole abdominal cavity and less inflammatory reaction secondary to the minimally invasive approach and sententiously dissection. And in order to preserving the function of lungs and heart, we tried to keep the position of the old patients in the horizon, not as the young patients keep in trendelenburg position during the LA operation. It is well known that LA is associated with less tissue trauma, shorter wound healing time, less rate of surgical site infection and shorter hospitalization time. The same results were also shown in our series. The earlier ambulation also could promote the patients' gastrointestinal peristalsis and prevention of the occurrence of accretion pneumonia. And the rate of

\section{References}

1. Cai YL, Xiong XZ, Wu SJ, Cheng Y, Lu J, Zhang J, Lin YX, Cheng NS. Single-incision laparoscopic appendectomy vs. conventional laparoscopic appendectomy: systematic review and meta-analysis. World J Gastroenterol 2013; 19: 5165-5173.

2. Addiss DG, Shaffer N, Fowler BS, Tauxe RV. The epidemiology of appendicitis and appendectomy in the United States. Am J Epidemiol 1990; 132: 910-925.

3. Segev L, Keidar A, Schrier I, Rayman S, Wasserberg N, Sadot E. Acute appendicitis in the elderly in the twentyfirst century. J Gastrointest Surg 2015; 19: 730-735.

4. Semm K. Endoscopic appendectomy. Endoscopy 1983; 15: 59-64.

5. Bozkurt MA, Unsal MG, Kapan S, Gonenc M, Dogan M, Kalayci MU, Alis H. Is laparoscopic appendectomy going to be standard procedure for acute appendicitis; a 5-year single center experience with 1,788 patients. Eur J Trauma Emerg Surg 2015; 41: 87-89.

6. Chan L, Shin LK, Pai RK, Jeffrey RB. Pathologic continuum of acute appendicitis: sonographic findings and 
clinical management implications. Ultrasound Q 2011; 27: 71-79.

7. Kapischke M, Friedrich F, Hedderich J, Schulz T, Caliebe A. Laparoscopic versus open appendectomy-quality of life 7 years after surgery. Langenbecks Arch Surg 2011; 396: 69-75.

8. Ortega AE, Hunter JG, Peters JH, Swanstrom LL, Schirmer B. A prospective, randomized comparison of laparoscopic appendectomy with open appendectomy. Laparoscopic Appendectomy Study Group. Am J Surg 1995; 169: 208-212.

9. Reshef A, Hull TL, Kiran RP. Risk of adhesive obstruction after colorectal surgery: the benefits of the minimally invasive approach may extend well beyond the perioperative period. Surg Endosc 2013; 27: 1717-1720.

10. Tsao KJ, St Peter SD, Valusek PA, Keckler SJ, Sharp S, Holcomb GW, 3rd, Snyder CL, Ostlie DJ. Adhesive small bowel obstruction after appendectomy in children: comparison between the laparoscopic and open approach. J Pediatr Surg 2007; 42: 939-942.

\section{*Correspondence to}

Tao Jiang

Department of General Surgery

Dongzhimen Hospital

The First Affiliated Hospital of Beijing University of Chinese Medicine

PR China

Xiaoguang Shi

Department of General Surgery

Dongzhimen Hospital

The First Affiliated Hospital of Beijing University of Chinese Medicine

PR China 